\title{
Application of Nursing Quality Improvement to Reduce the Incidence of Hypoglycemia During Colonoscopy Procedure in Diabetes Patients
}

\author{
Yan Zhou ${ }^{\dagger}$, Wane Zhao ${ }^{\dagger}$, Xiaohua Lu*, Jiewei Huang, Qinglin Chen, Yan Liu, Xixi Luo \\ Department of Endocrinology, The First Affiliated Hospital of Jinan University, Guangzhou, China \\ Email address: \\ 992054881@qq.com (Yan Zhou),223644054@qq.com (Wane Zhao),536414169@qq.foxmail.com (Xiaohua Lu), \\ chgzhjw@sohu.com (Huang Jiewei), 408783096@qq.com (Qinglin Chen), 904258141@qq.com (Yan Liu), \\ 2534105932@@q.com (Xixi Luo) \\ *Corresponding author \\ †Yan Zhou and Wane Zhao are co-first authors.
}

\section{To cite this article:}

Yan Zhou, Wane Zhao, Xiaohua Lu, Jiewei Huang, Qinglin Chen, Yan Liu, Xixi Luo. Application of Nursing Quality Improvement to Reduce the Incidence of Hypoglycemia During Colonoscopy Procedure in Diabetes Patients. American Journal of Nursing Science. Vol. 9, No. 3, 2020, pp. 149-154. doi: 10.11648/j.ajns.20200903.23

Received: April 12, 2020; Accepted: May 3, 2020; Published: May 14, 2020

\begin{abstract}
Objective: To explore the effect of Nursing Quality Improvement on reducing the incidence of hypoglycemia prior to and during colonoscopy in diabetes patients. Methods: The causes of hypoglycemia prior to and during colonoscopy were first analyzed. Then, the corresponding improvement plan and special procedure rules prior to and during colonoscopy were put forward. All the nurses including 23 nurses in the endocrinology department and 2 nurses in the endoscopy room must strictly obey the procedure rules. The Ward of Manager and the Nurse in charge were responsible for supervision. The awareness rate of nurse staff on the knowledge of colonoscopy and behavior improvement of nurse staff before and after the improvement were compared. The incidence of hypoglycemia prior to and during colonoscopy before and after the improvement were calculated. Results: After the Nursing Quality Improvement, the awareness rate of colonoscopy related knowledge in nurse staff was improved from 97.6 to $100 \%$. All the nurses changed their nursing behavior related to bowel preparation from "Occasionally" to "Always". Besides, the incidence of hypoglycemia prior to and during colonoscopy was reduced from $25 \%$ to $8.58 \%$ after the improvement. Conclusion: The application of Nursing Quality Improvement prior to and during colonoscopy can reduce the incidence of hypoglycemia and ensure the safety of patients.
\end{abstract}

Keywords: Nursing Quality Improvement, Incidence of Hypoglycemia, Colonoscopy

\section{Introduction}

Diabetes mellitus (DM) is an endocrine and metabolic disease characterized by high blood sugar, which is caused by many pathogenic factors such as heredity, environment, and lifestyle. In 2010 and 2013, the prevalence of diabetes in the population over 18 years old in China was $9.7 \%$ and $10.4 \%$ respectively [1]. Some patients with diabetes need to have colonoscopy during hospitalization. Hypoglycemia is a common complication in bowel preparation, with a reported incidence of $1.5 \%-13.5 \%$ [2-3]. For those patients who were old or weak or with many basic diseases, hypoglycemia occurred more easily because of less food intake, longer fasting time, anxiety, tension and other psychological conditions [4]. In this study, the incidence of hypoglycemia prior to and during colonoscopy in hospitalized diabetes patients of our hospital was investigated from January to June in 2016. It was found that hypoglycemia occurred in 10 patients of 40 diabetes patients, and the incidence of hypoglycemia was 25\%. Therefore, since July 2016, Nursing Quality Improvement was carried out for the bowel preparation of hospitalized diabetes patients who took colonoscopy. Compared with the incidence of hypoglycemia before the improvement, good result was achieved. There has been no similar reports abroad. The summary report is as follows. 


\section{Data and Methods}

\subsection{Research Objects}

Patients who underwent hypoglycemia from January 2016 to December 2019 were selected as the study subjects. Members of the quality improvement team were composed of Ward of Manager, Nurse in charge, Registered Nurses, Enrolled Nurses and Registered Nurses of endoscopy room. In the team, there were 23 nurses in the endocrinology department and 2 nurses in the endoscopy room. The age of nurses was 26-58 years, with an average of $36.40+9.53$ years; the working age was 3-30 years, with an average of $15.24+$ 8.55 years, including 3 Master degree and 12 College degree.

\subsection{Research Methods}

\subsubsection{Establishment of the Quality Improvement Group}

The quality improvement group was established in June 2016. It was composed of the Ward of Manager of Department of Endocrinolog, Nurse in charge, Registered Nurses, Enrolled Nurses, endocrinologists, and the Registered Nurses of endoscopy room. The Ward of Manager of the Endocrinology Department was the team leader, and the Nurse in charge was the person who was in charge of the organization and distribution of the whole group activities.

\subsubsection{Data Collection}

The incidence of hypoglycemia in diabetic patients undergoing colonoscopy was statistically analyzed by using the registration form and nursing form of hypoglycemia in diabetic patients. From January 2016 to June 2016, there were 40 inpatients of diabetes mellitus prior to and during colonoscopy. Ten of them developed hypoglycemia, and the incidence of hypoglycemia was $25 \%$.

\subsubsection{Activity Schedule}

The whole activity stages included selecting the theme, planning and grasping the current situation, setting the objectives, analyzing the causes, formulating the countermeasures, implementing the countermeasures, inspecting the effects, formulating the consolidation measures, evaluating, improving, and summarizing the materials as well as releasing the results. The activities of each stage were summarized and evaluated at regular time.

\subsubsection{Set up the Objective}

We analyzed the key improvement factors of the project through Pareto Diagram and adopted the target value calculation method of quality control circle. The improvement range $=$ Plato's key points to be improved $\times$ expected improvement ability. Improvement rate $=70 \% \times 90 \%=63 \%$. Target value $=$ actual value before improvement $\times(1$-expected improvement range). Through calculation, the target value $=$ $25 \% \times(1-63 \%)=9.25 \%$. That is to say, the incidence of hypoglycemia in diabetic inpatients would be reduced from $25 \%$ to $9.25 \%$ before improvement by December 30 of 2016 .

\subsubsection{Cause Analysis}

Brainstorming and fishbone diagram (cause and effect diagram) [5] were used to find various causes. We analyzed it from the aspects of people, things, law, environment, etc. (1) From the aspect of human, the reasons were as follows: for the nurses, they did not pay attention to the adjustment of hypoglycemic program and the evaluation of hypoglycemia prior to and during colonoscopy. They were also lack of diet education and training; for the patients, the causes were their change of diet, the lack of intake, the lack of knowledge about hypoglycemia, the lack of vision on the importance of hypoglycemia, and the lack of awareness of potential risks; for the doctors, the causes were their lack of timely adjustment of insulin and hypoglycemic drug dosage as well as lack of knowledge and attention on bowel preparation. (2) From the aspect of material analysis: We were lacking in the graphic data and intestinal preparation guidance was not detailed and comprehensive. (3) From the perspective of law, we were lacked of standardized guidelines during the preparation of endoscopy for diabetic patients. We were also lack of standardized procedures and systems as well as regular communication with people in the endoscopy room. Besides, people in the endoscopy room were not clear about diabetic patients. (4) From the aspect of the environment, there were too many patients undergoing colonoscopy. This led to the inconsistent waiting time of diabetic patients during colonoscopy.

\subsubsection{Development and Implement of Measures}

\section{(i). Medical Training}

Under the guidance of two specialized diabetes nurses, the head nurse was responsible for strengthening the quality control. The training contents included: (1) the correct assessment method of blood glucose during the endoscopy of in-patients with diabetes mellitus; (2) the correct assessment method of diet during the inspection of in-patients with diabetes mellitus; (3) the health education of knowledge related to intestinal cleaning preparation for in-patients with diabetes mellitus; (4) the ability to carry out prospective prevention and treatment of possible risks prior to and during colonoscopy. Through training, the awareness of prevention of hypoglycemia was improved prior to and during colonoscopy, which would reduce the incidence of hypoglycemia.

\section{(ii). Patient Education}

Before colonoscopy, health education such as information about blood sugar and diet was given to patients. The contents of education included how to choose diet during intestinal preparation, the timing of blood glucose measurement, intestinal cleaning preparation, and the importance of preventing hypoglycemia during intestinal preparation. Besides, the nurse in charge should carry out key education according to the precautions on the appointment form.

\section{(iii). Specific Implementation Guidelines}

(1) The Enrolled Nurse reviewed doctors' order of colonoscopy. Then the Enrolled Nurse marked and sealed the colonoscopy examination sheet with red pen: diabetic patients. After we communicated with the 
endoscopy center, the endoscopy room would arrange to do the colonoscopy in the morning. At the same time, they also sealed on the colonoscopy appointment form: diabetic patients. After that, the endoscopy room would give priority to arrange the examination of diabetic patients after receiving the appointment form, so as to shorten the waiting time.

(2) After receiving the colonoscopy appointment form, the Enrolled Nurse shall inform the Registered Nurse.

(3) The Registered Nurse checked the date of the patient's colonoscopy and the hypoglycemic plan. Then, the nurse informed the doctor of the day of conducting colonoscopy for the patient and communicated with the doctor about whether the treatment plan of the patient needed to be adjusted.

(4) After receiving the notice, the Enrolled Nurse would inform the patient about the knowledge of colonoscopy and the method of intestinal preparation. The nurses would also assist the patient to order meals.

(5) The Registered Nurse checked the food preparation of the patient (pure milk, porridge, drinks, etc.). We would inform the patient to take a low-fat, soft, and half liquid diet with less dregs (porridge, soup powder, and soup noodles) a day before the examination. Besides, we would instruct the patient to eat less and more meals, increase 4-5 meals per day, and adjust the treatment plan in time according to the amount of food.

(6) The Registered Nurse teached the patient how to recognize hypoglycemia and how to deal with it. During the period of colonoscopy, the frequency of blood glucose monitoring (colonoscopy preparation day and examination day) should be increased. Blood glucose should be measured before three meals, after three meals, before bedtime, and at 3:00 in the morning. If the blood glucose before bedtime of exercise was lower than $5.6 \mathrm{mmol} / \mathrm{L}$, the patient should be instructed to eat to prevent hypoglycemia. Strenuous exercise should be avoided during the period of colonoscopy preparation.

(7) On the day of examination, if the colonoscopy was checked in the morning, Polyethylene Glycol Electrolytes Powder was given orally and fasted for breakfast at 5:00 in the morning. If the colonoscopy was checked in the afternoon, Polyethylene Glycol Electrolytes Powder was given at 11:00 in the morning and the patient could eat liquid food (milk, porridge water) for breakfast.

(8) Blood glucose was measured before colonoscopy to ensure that the blood glucose was within the safe range before colonoscopy.

(9) Blood glucose should be measured in time after colonoscopy. For some patients who have polypectomy in colonoscopy, half fluid diet was still needed for one week after operation. However, hypoglycemia may still occur. Therefore, the frequency of blood glucose measurement should be continued to increase.

\subsubsection{Check the Occurrence of Hypoglycemia with Diabetic Colonoscopy and Improve It in Time}

We established a register of colonoscopy in the department. The record of hypoglycemia during colonoscopy was registered by the nurse in charge who would understand the symptoms and treatment process of hypoglycemia during intestinal preparation. The implementation of measures should be checked to reduce hypoglycemia in diabetic patients undergoing colonoscopy weekly and monthly by the nursing team leader and the head nurse. We would record the problems in the implementation process, put forward the improvement of objectives and measures, make new plans again, and implement a new round of continuous quality improvement management [6-7]. The nursing workflow chart of diabetic colonoscopy (as shown in the figure below) was formed to gradually reduce the incidence of hypoglycemia.

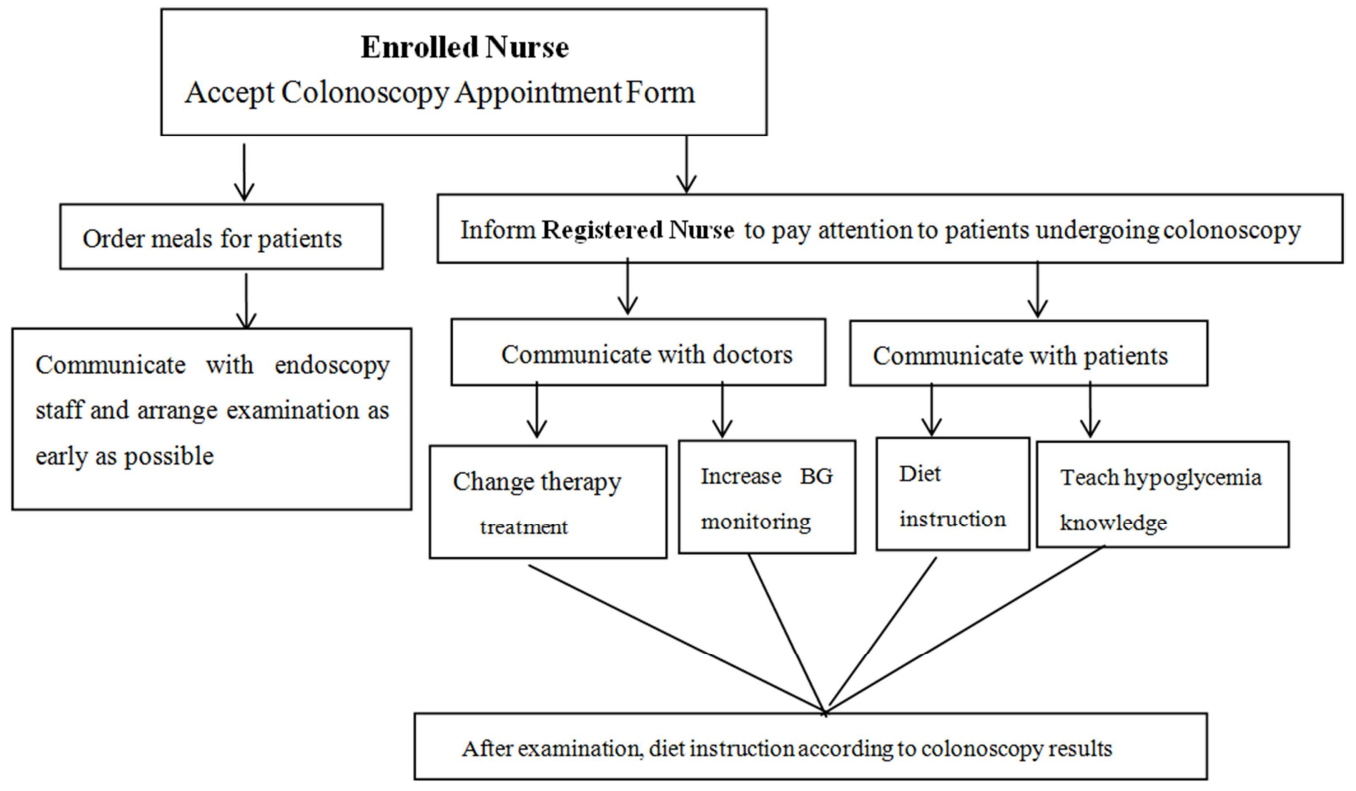

Figure 1. Nursing workflow chart of diabetic colonoscopy. 


\subsection{Statistical Analysis}

SPSS16.0 software was used for statistical analysis. The measurement data were expressed as $(\mathrm{x} \pm \mathrm{s})$ by $\mathrm{t}$-test, and the counting data were tested by $\chi 2$ test. $P<0.05$ indicated that the difference was statistically significant.

\section{Result}

\subsection{Comparison of the Awareness Rate of Nurse Staff on the Knowledge of Colonoscopy in Hospitalized Diabetes Patients Before and After the Improvement}

The results of questionnaire showed that before the implementation of quality improvement, the average awareness rate of 25 nurse staff was $97.6 \%$. After the nurse quality improvement, the awareness rate was $100 \%$.

\subsection{Comparison of the Behavior Improvement of Nurse Staff on Hospitalized Diabetes Patients Undergoing Colonoscopy}

After the nursing quality improvement, 25 nurses changed their behavior from "Occasionally" to "Always" during bowel preparation for hospitalized diabetes patients undergoing colonoscopy. (Table 1)

Always means that the frequency of relevant behaviors in nurse staff was more than 7 times out of every ten times. Occasionally means that the frequency of relevant behaviors in nurse staff was less than 2 times out of every ten times

Table 1. Comparison of the behavior improvement of nurse staff on hospitalized diabetes patients undergoing colonoscopy.

\begin{tabular}{|c|c|c|c|c|c|c|c|c|}
\hline \multirow{2}{*}{ Items } & \multicolumn{2}{|l|}{ Always } & \multirow{2}{*}{$\chi^{2}$} & \multirow{2}{*}{$p$} & \multicolumn{2}{|c|}{ Occasionally } & \multirow{2}{*}{$\chi^{2}$} & \multirow{2}{*}{$p$} \\
\hline & Before & After & & & Before & After & & \\
\hline Tell hypoglycemia knowledge & $17(68.0)$ & $25(100.0)$ & 7.292 & 0.007 & $8(32.0)$ & $0(0.0)$ & 7.292 & 0.007 \\
\hline Test midnight BG & $14(56.0)$ & $25(100.0)$ & 14.103 & 0.000 & $11(44.0)$ & $0(0.0)$ & 14.103 & 0.000 \\
\hline Test BG before examination & $11(44.0)$ & $24(96.0)$ & 16.095 & 0.000 & $14(56.0)$ & $1(4.0)$ & 16.095 & 0.000 \\
\hline Test BG after examination & $11(44.0)$ & $25(100.0)$ & 19.444 & 0.000 & $14(56.0)$ & $0(0.0)$ & 19.444 & 0.000 \\
\hline Diet instruction & $22(88.0)$ & $25(100.0)$ & 1.418 & 0.234 & $3(12.0)$ & $0(0.0)$ & 1.418 & 0.234 \\
\hline Focus patient appetite & $19(76.0)$ & $25(100.0)$ & 4.735 & 0.030 & $6(24.0)$ & $0(0.0)$ & 4.735 & 0.030 \\
\hline Communicate plan with doctor & $18(72.0)$ & $25(100.0)$ & 5.980 & 0.014 & $7(28.0)$ & $0(0.0)$ & 5.980 & 0.014 \\
\hline Check food preparation of patient & $16(64.0)$ & $25(100.0)$ & 8.672 & 0.003 & $9(36.0)$ & $0(0.0)$ & 8.672 & 0.003 \\
\hline
\end{tabular}

\subsection{Comparison of the Incidence of Hypoglycemia in Diabetes Patients Before and After the Improvement}

The total average incidence of hypoglycemia after the improvement (8.58\%) was lower than that before the improvement $(25 \%)$, and the difference was statistically significant $(\mathrm{P}<0.001)$. (Table 2$)$

Table 2. Comparison of the incidence of hypoglycemia in diabetes patients undergoing colonoscopy before and after the improvement.

\begin{tabular}{lllllll}
\hline & Year & Total cases & Hypoglycemia cases & Incidence of Hypoglycemia (\%) & $\chi^{2}$ & $\boldsymbol{p}$ \\
\hline Before & $2016.1-2016.6$ & 40 & 10 & 25.0 & 5.559 & 0.018 \\
After & $2016.7-2016.12$ & 62 & 5 & 8.06 & 9.252 & 0.002 \\
& $2017.1-2017.12$ & 138 & 9 & 6.52 & 4.366 & 0.037 \\
& $2018.1-2018.12$ & 122 & 14 & 11.48 & 6.384 & 0.012 \\
& $2019.1-2019.12$ & 121 & 10 & 8.26 & 9.296 & 0.002 \\
\hline
\end{tabular}

\section{Discussion}

Diabetes mellitus is a common chronic disease in China. Hypoglycemia is one of the most common acute complications of diabetes. If the blood glucose is lower than $3.9 \mathrm{mmol} / \mathrm{L}$ when patients receive the drug treatment, it belongs to the category of hypoglycemia [1]. Hypoglycemia is also a potential serious complication of diabetes, which is far more harmful than hyperglycemia. This is because that continuous and severe hypoglycemia can lead to brain dysfunction, increase the risk of cardiovascular and cerebrovascular accidents, and even lead to death. Most type 1 diabetes patients cannot avoid hypoglycemia, which occur thousands of times in a lifetime. The incidence of hypoglycemia in type 2 diabetes patients is lower than that in type 1 diabetes [8]. Yang Cunmei's study found that the incidence of hypoglycemia in diabetes inpatients was $1.29 \%$, among which 317 cases developed severe hypoglycemia and the incidence was $0.29 \%$ [9]. A severe hypoglycemia which could cause cardiovascular events may offset the benefits of maintaining blood glucose in the normal range for a lifetime.

In this study, the incidence of hypoglycemia prior to and during colonoscopy in hospitalized diabetes patients of our hospital was investigated from January to June in 2016. It was found that hypoglycemia occurred in 10 patients of 40 diabetes patients, and the incidence of hypoglycemia was $25 \%$. Because of the high incidence of hypoglycemia, the Nursing Quality Improvement must be carried to reduce the incidence of hypoglycemia. Our Nursing Quality Improvement included 
establishing the Quality Improvement Group, analyzing the causes of hypoglycemia prior to and during colonoscopy, putting forward the corresponding improvement plan and special procedure rules, and developing the flow chart of colonoscopy for diabetic patients during colonoscopy procedure. All the nurses must strictly obey the procedure rules. The implementation of the improvement should be checked weekly and monthly by the Ward of Manager and the Nurse in charge. We also recorded the problems in the implementation process, set the new goal and made new plans again, and then implemented a new round of nursing quality improvement.

Therefore, since July 2016, Nursing Quality Improvement was carried out for the bowel preparation of hospitalized diabetes patients who took colonoscopy. The results in this study show that before the implementation of Nursing Quality Improvement, the average awareness rate of 25 nurse staff was $97.6 \%$ on drug preparation, diet requirements, intestinal cleanliness requirements, hypoglycemia symptoms during the preparation of colonoscopy and hypoglycemia treatment during the preparation of colonoscopy. After the quality improvement, the awareness rate of colonoscopy related knowledge was $100 \%$. In terms of behavior related to colonoscopy, just before the quality improvement, 11 nurses occasionally measured blood sugar in the early morning of the day of colonoscopy; 12 nurses occasionally measured blood sugar before or after colonoscopy; 6 nurses occasionally communicated the medical treatment plan with doctors; 6 nurses never looked at the treatment plan during colonoscopy. All of these indicated that we lacked attention to hypoglycemia prior to or during colonoscopy, and there was no unified process and guidance. Therefore, we need to improve the quality of related projects [10-12] After improvement, 25 nurses changed their behavior to "Always" during bowel preparation, which became part of their normal daily work. With this study, we know that during colonoscopy preparation in diabetes patients, nurse staff not only need to master the relevant knowledge of colonoscopy, but also need to increase the frequency of blood glucose monitoring, pay attention to the treatment plan of patients, and effectively communicate the medication plan with doctors according to the established norms and procedures.

The results of Table 2 showed that after six months of the Nursing Quality Improvement, the average incidence of hypoglycemia from July to December in 2016 was $8.06 \%$. After over three years of continuous follow-up, the average incidence of hypoglycemia was $8.58 \%$ from July 2016 to December 2019 after the improvement. All of the average incidences reached the target value of $9.25 \%$ or less. But in 2018, we found that the hypoglycemia rate rebounded. Because of the employment of many new nurses in 2018 and insufficient training for the new staff, the incidence of hypoglycemia in 2018 was $11.48 \%$. So, in 2019, many relevant trainings for new nurses were carried [13]. After that, the incidence of hypoglycemia was reduced to $8.26 \%$ during 2019. We conclude that the Nursing Quality Improvement has a positive effect on reducing hypoglycemia prior to and during colonoscopy

In short, the application of Nursing Quality Improvement prior to and during colonoscopy can reduce the incidence of hypoglycemia and ensure the safety of patients. Therefore, this Nursing Quality Improvement could be recommended for all diabetes patients undergoing colonoscopy. However, the Nursing Quality Improvement in this study is mainly for hospitalized diabetic patients. Therefore, further research is needed for those diabetic outpatients undergoing colonoscopy in the department, which is the deficiency of this study.

\section{Ethics Approval and Consent to Participate}

Not applicable.

\section{Consent for Publication}

Not applicable.

\section{Competing Interests}

The authors declare that they have no competing interests.

\section{Fund}

Guangdong Medical Research Fund, Grant Number: A2020574.

\section{References}

[1] Guidelines for the prevention and treatment of type 2 diabetes in China (2017 Edition) [J]. Chinese Journal of Practical Medicine, 2018, 38 (04): 292-344.

[2] Zhang Huiying, Zheng Rou, Sun Siwen, et. al. Safety of intestinal preparation by enteroscopy in 2786 elderly patients with diabetes mellitus. [J]. Chinese Journal of Gerontology, 2015, 35 (24): 7229-7230.

[3] Lv Minfang, Zheng Jingjing, Zheng Qiongna, et. al. Analysis of blood glucose monitoring in intestinal preparation of non diabetic elderly patients [J]. Nursing and rehabilitation, 2015, 09 (24): 852-853.

[4] You Zhao yuan, Liu Juan, Fang tianlu. Nursing strategy of preventing hypoglycemia in patients with type 2 diabetes during peri endoscopic examination [J]. Journal of nursing training, 2013, 28 (13): 1189-1190.

[5] Liang Hongkuan, Luo Ai, Xv Xiaojia, et. al.. Application of continuous quality improvement in reducing the incidence of hypoglycemia in diabetic inpatients. [Jodern medicine and health, 2020, 36 (04): 601-604.

[6] Hopper Ingrid, Easton Kellie, Bader Illona, Campbell James, Busija Lucy, Markey Peter, Bergin Peter, Kaye David. Impact of a model of care for heart failure in-patients to reduce variation in care - a quality improvement project. [J]. Internal medicine journal, 2020. 
[7] Mate Kedar S, Rakover Jeffrey, Cordiner Kay, Noble Amy, Hassan Noura. Novel quality improvement method to reduce cost while improving the quality of patient care: retrospective observational study. [J]. BMJ quality \& safety, 2020.

[8] Endocrinology branch of Chinese Medical Association, Expert consensus on hypoglycemia management of Chinese diabetic patients [J]. Chinese Journal of Endocrinology and metabolism, 2012, 28 (8): 619-623.

[9] Yang Cunmei, Ma Yanlan, Kang Jun, et. al. Investigation and analysis of the time period of hypoglycemia in diabetic inpatients $[\mathrm{J}]$. Chinese Journal of nursing, 2015, 30 (3): 303-307.

[10] Deng Wanping, Chen Bing, He Xiaohua, et. al. Continuous quality improvement based on the analysis of the causes of hypoglycemia in hospitalized diabetic patients (Application of safety management) $[\mathrm{J}]$. Electronic Journal of practical clinical nursing, 2019, 4 (38): 175.

[11] Papa Frank Joseph, Alexander Jerry H. Aggregated student confidence estimates support continuous quality improvements in a competencies-oriented curriculum. [J]. BMJ open quality, $2019,8(1)$

[12] Lum Brandon, Png Hong Muay, Yap Hock Lan, Tan Cindy, Sun Bixian, Law Yen Hoon. Streamlining workflows and redesigning job roles in the theatre sterile surgical unit. [J]. BMJ open quality, 2019, 8 (3).

[13] Judith A. Halstead. Fostering Innovation in Nursing Education: The Role of Accreditation [J]. Teaching and Learning in Nursing, 2020, 15 (1). 